ISBN 978-93-84468-83-5

International Conference on Studies in Arts, Law and Education

(ICALE-2016)

Singapore Dec. 5-6, 2016

\title{
Factors of Storage Management Performance of Pearl Bubble Milk Tea Business, Bangkok
}

\author{
Patchaploy Jareantanyachod, and Chinnaso Visitnitikija \\ chinnaso12@gmail.com \\ Post Graduate School of business administration \\ Kasembundit University, Bangkok
}

\begin{abstract}
The goal of this study was to determine the factors of storage management performance of pearl bubble milk tea business. Samples were 135 pearl bubble milk tea business entrepreneur and their employees by using a check list and rating scale questionnaire. The statistics used for data analysis were percentage, mean, standard deviation, T-test, One-way ANOVA, and regression analysis. On the basis of the results of this study, it can be concluded that most of respondents were females, age between 31-35 years old, bachelor degree graduated, business owners, and a period of 3-5 years' work experience. The average picture of opinions on the factors of storage management performance of pearl bubble milk tea business were in the medium level, such as products quality, accurate stock detail, precise items list, adequate stock level, competent personnel, prompt service, reasonable work area floor plan, ABC inventory storage, appropriated stock level, on time delivery, dependable moving equipment, temperature controlled storage, technology management, successful designed transport routine, respectively. The hypothesis testing revealed that the management of cost, dead stock preventive, just in time, speed, supply, moving equipment, $A B C$ inventory storage, dependability, and technology had relation to the factors of storage management performance of pearl bubble milk tea business at statistical significant 0.05 level. Recommendations from this study were administrative officers or business entrepreneur should emphasis on cost, demand, supply, just in time management, promptness, appropriate moving equipment, ABC inventory storage management, dependability, and technology.
\end{abstract}

Keywords----- storage management, bubble milk, bubble tea, business

\section{Introduction}

Tea is one of most favorite beverages which has caffeine in it. Not only relief of thirsty and sleepy, but it is good for your health because there is antioxidants in tea which reduce chlorophyll that prevent cancer and heart disease.

Pearl milk tea originated from Taiwan which classified as a beverage made from tea and add flavor, color, and pearl. When drinking milk tea with pearl together it bring out the pleasurable taste and become favorite drink of people of all ages.

The beverages business currently is still an interesting business. Pearl milk tea business in particular, which is very popular in Thai community and this drink can be sold all day long because of hot climate of the country. Pearl milk tea products come into play in a role of everyday life on any ages. As for distribution channel, the pearl milk tea stores are offered everywhere, such as BTS / MRT stations, department stores, schools, universities, offices buildings, etc. The pearl milk tea has become competitive of the business and result in the expanding greatly of pearl milk tea franchising. There are a wide variety of pearl milk tea business market with different brands occurs continuously everywhere, such as kiosk, vending counter, and vending cart. Each of the pearl milk tea brands have different flavor, prices, production process, etc. 
Because of greatly competitive pearl milk tea business, to be more advantages to the other the competitor, they have to create more variety of products than the other to attract more consumer to choose their brands, such as red tea, green tea, and Ulong tea with more flavor in it, and add a variety of tastes, such as Taro milk tea, Strawberry milk tea, etc., on top of that they put the toping, such as Jelly, pudding, and grass jelly. Some stores, even add coffee drinks in their menu also. Because of high competitive in the business, consumer has more option of products to choose from, so the service after sale are needed such as suggesting products and ordering raw materials with quality of the services in order to meet consumer requirement.

From currently popular of pearl milk tea, there is opportunity for a new entrepreneur to invest into this business of franchising to begin with, because franchisor will provided turn key business that the new owner will aim for the success of the investment.

The popularity of pearl milk tea business, the business operators must come across the high competitive market. Therefore, the approach to compete with competitors by using a logistics strategy in order to survive from the business in the competitive market conditions. Most of companies, government agencies, and all businesses are not aware of the logistics issue that can reduce the cost of business operations, no different from quality control and reduction of waste in the production business as well. The logistics activity in pearl milk tea business, such as operating cost reduction, operation with speed, reliability, and quality of products.

From the examined the condition of the pearl milk tea business, it could be concluded that there was no effective warehouse inventory system and the problems founded, such as inventories were not accurately met, the resulted from that was shorted of raw material supplies, late delivery, higher cost of raw material supplies, and wasted warehouse storage space, and this caused by not having to plan an effective management strategy.

Logistics is generally the detailed organization and implementation of a complex operation. In a general business sense, logistics is the management of the flow of things between the point of origin and the point of consumption in order to meet requirements of customers or corporations. The resources managed in logistics can include physical items such as food, materials, animals, equipment, and liquids; as well as abstract items, such as time and information. The logistics of physical items usually involves the integration of information flow, material handling, production, packaging, inventory, transportation, warehousing, and often security.

Logistics management is the part of supply chain management that plans, implements, and controls the efficient, effective forward, and reverse flow and storage of goods, services, and related information between the point of origin and the point of consumption in order to meet customer's requirements. The complexity of logistics can be modeled, analyzed, visualized, and optimized by dedicated simulation software. The minimization of the use of resources is a common motivation in all logistics fields. A professional working in the field of logistics management is called a logistician.

Warehouse management system and Warehouse control system: Although there is some overlap in functionality, warehouse management systems (WMS) can differ significantly from warehouse control systems (WCS). Simply put, a WMS plans a weekly activity forecast based on such factors as statistics and trends, whereas a WCS acts like a floor supervisor, working in real time to get the job done by the most effective means. For instance, a WMS can tell the system that it is going to need five of stock-keeping unit (SKU) A and five of SKU B hours in advance, but by the time it acts, other considerations may have come into play or there could be a logjam on a conveyor. A WCS can prevent that problem by working in real time and adapting to the situation by making a last-minute decision based on current activity and operational status. Working synergistically, WMS and WCS can resolve these issues and maximize efficiency for companies that rely on the effective operation of their warehouse or distribution center.

Logistics activities and fields: Inbound logistics is one of the primary processes of logistics, concentrating on purchasing and arranging the inbound movement of materials, parts, and/or finished inventory from suppliers to manufacturing or assembly plants, warehouses, or retail stores. Outbound logistics is the process related to the storage and movement of the final product and the related information flows from the end of the production line to the end user.

Given the services performed by logisticians, the main fields of logistics can be broken down as follows:

Procurement logistics consists of activities such as market research, requirements planning, make-or-buy decisions, supplier management, ordering, and order controlling. The targets in procurement logistics might be contradictory: maximizing efficiency by concentrating on core competences, outsourcing while maintaining the autonomy of the company, or minimizing procurement costs while maximizing security within the supply process. 
Distribution logistics has, as main tasks, the delivery of the finished products to the customer. It consists of order processing, warehousing, and transportation. Distribution logistics is necessary because the time, place, and quantity of production differs with the time, place, and quantity of consumption.

Disposal logistics has as its main function to reduce logistics $\operatorname{cost}(\mathrm{s})$ and enhance service(s) related to the disposal of waste produced during the operation of a business.

Reverse logistics denotes all those operations related to the reuse of products and materials. The reverse logistics process includes the management and the sale of surpluses, as well as products being returned to vendors from buyers. Reverse logistics stands for all operations related to the reuse of products and materials. It is "the process of planning, implementing, and controlling the efficient, cost effective flow of raw materials, inprocess inventory, finished goods and related information from the point of consumption to the point of origin for the purpose of recapturing value or proper disposal. More precisely, reverse logistics is the process of moving goods from their typical final destination for the purpose of capturing value, or proper disposal. The opposite of reverse logistics is forward logistics."

Green Logistics describes all attempts to measure and minimize the ecological impact of logistics activities. This includes all activities of the forward and reverse flows. This can be achieved through intermodal freight transport, path optimization, vehicle saturation and city logistics.

RAM Logistics combines both business logistics and military logistics since it is concerned with highly complicated technological systems for which Reliability, Availability and Maintainability are essential, ex: weapon systems and military supercomputers.

Asset Control Logistics: companies in the retail channels, both organized retailers and suppliers, often deploy assets required for the display, preservation, promotion of their products. Some examples are refrigerators, stands, display monitors, seasonal equipment, poster stands \& frames.

Emergency logistics is a term used by the logistics, supply chain, and manufacturing industries to denote specific time-critical modes of transport used to move goods or objects rapidly in the event of an emergency. The reason for enlisting emergency logistics services could be a production delay or anticipated production delay, or an urgent need for specialized equipment to prevent events such as aircraft being grounded (also known as "aircraft on ground"-AOG), ships being delayed, or telecommunications failure. Humanitarian logistics involves governments, the military, aid agencies, donors, non-governmental organizations and emergency logistics services are typically sourced from a specialist provider.

The term production logistics describes logistic processes within a value adding system. Production logistics aims to ensure that each machine and workstation receives the right product in the right quantity and quality at the right time. The concern is with production, testing, transportation, storage and supply. Production logistics can operate in existing as well as new plants: since manufacturing in an existing plant is a constantly changing process, machines are exchanged and new ones added, which gives the opportunity to improve the production logistics system accordingly. Production logistics provides the means to achieve customer response and capital efficiency. Production logistics becomes more important with decreasing batch sizes. In many industries (e.g. mobile phones), the short-term goal is a batch size of one, allowing even a single customer's demand to be fulfilled efficiently. Track and tracing, which is an essential part of production logistics due to product safety and reliability issues, is also gaining importance, especially in the automotive and medical industries.

Construction Logistics is known to mankind since ancient times. As the various human civilizations tried to build the best possible works of construction for living and protection. Now the construction logistics emerged as vital part of construction. In the past few years construction logistics has emerged as a different field of knowledge and study with in the subject of supply chain management and logistics.

From the introduction above, research is interested in studying the factors that affect the management of warehouse Pearl milk tea business in order to strengthen inventory management control system that is effective and appropriate for the model of the operation of Pearl milk tea a business.

\section{Study Results}

Findings indicated that most of 135 respondents were females, age 31-35 year old, bachelor degree graduated, entrepreneur, and work experiences 3-5 years. The overall picture of the opinions on factors of storage management affecting storage management efficiency of pearl bubble milk tea business were in high 
level in ascending order as followed: quality aspect, speed aspect, dependability aspect, and cost aspect from the following in details as shown on the figure.

Figure 1:

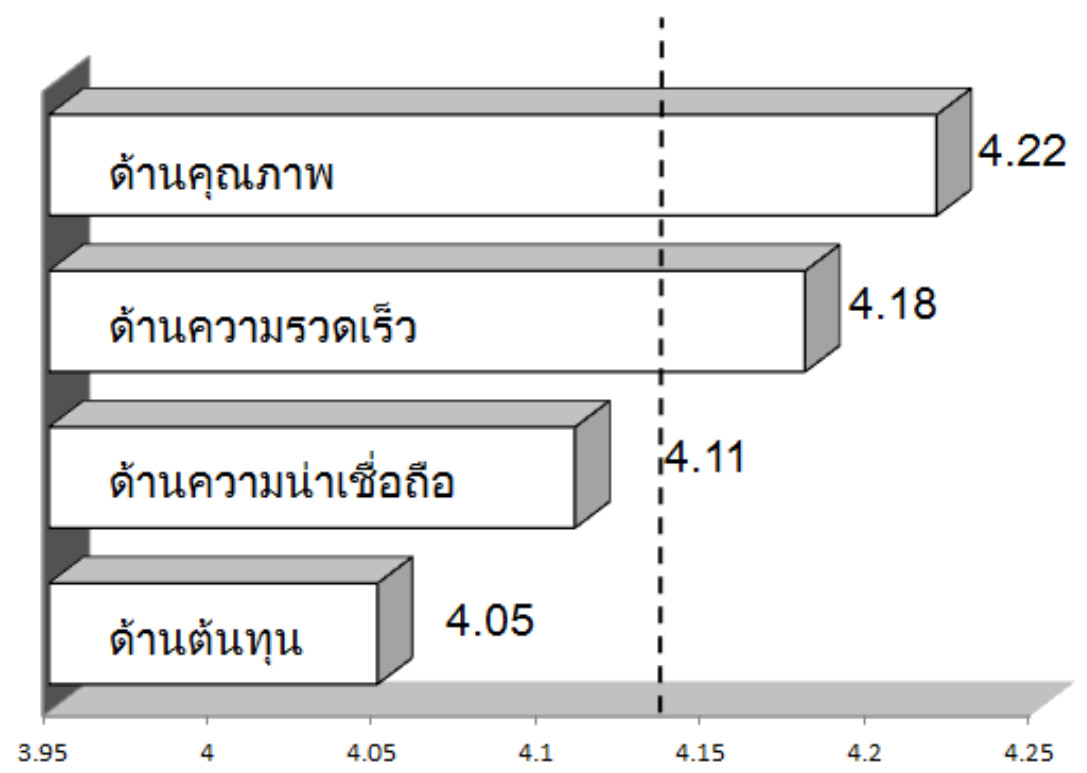

Fig. 1 shows factors affecting storage management efficiency of pearl bubble milk tea business consisted of 4 aspects, such as the efficiency of storage management of pearl bubble milk tea business was in medium level of the opinions as following detail

\section{Discussion}

Results from the study of the overall picture of the opinions on factors of storage management affecting storage management efficiency of pearl bubble milk tea business were in high level which supported by the research of Chutchaval Amartayakul. (2545 B.E.).

Cost of warehouse management of pearl bubble milk tea business revealed that the overall picture of the opinions such as cost of business management were in high level which supported by the research of Vasana Jaroonsrichotikumjon. (2550 B.E.).

Speed of warehouse management of pearl bubble milk tea business revealed that the overall picture of the opinions such as speed of storage management were in high level which supported by Nuruemon Sanjaivong. (2558 B.E.).

Dependability of warehouse management of pearl bubble milk tea business revealed that the overall picture of the opinions such as dependability of storage management were in high level which supported by Varagorn Thongeng. (2551 B.E.).

Quality of warehouse management of pearl bubble milk tea business revealed that the overall picture of the opinions such as quality of storage management were in highest level which supported by Chinnaso Visitnitikija. (2551 B.E.).

The efficiency of warehouse management of pearl bubble milk tea business revealed that the overall picture of the opinions such as the efficiency of storage management were in medium level which supported by Kanpanee Buntavi. (2557 B.E.).

The factors of personal different affected the efficiency of storage management of pearl bubble milk tea business.

\section{Recommendations from the Study}

Recommendations from the study of factors of storage management affecting storage management efficiency of pearl bubble milk tea business were that store owners should emphasize on cost of running the business, manage inventory, just in time management, speedy management, stock inventory, appropriate 
working equipment, inventory with application technology, quality management, and well maintain quality of the products.

\section{References}

[1] Kanpanee Buntavi. (2557 B.E.). Service quality of Laem Chabang Port. Independent Study. Master of Business Administration, Kasem Bundit University.

[2] Jerawan Sutilak \& Pinya Silayoi. (2551 B.E.). Work efficiency development of employees of Plastic container industry at Thai foods industry. The $46^{\text {th }}$ academic conference of Kasetsart University: Agricultural industry department. (Page 41-48). Bangkok. Kasetsart University Printing.

[3] Chutchaval Amartayakul. (2545 B.E.). Management development of animal foods warehouse, Master of Engineering. Faculty of Engineering. King Mongkut's University of technology Thonburi.

[4] Chinnaso Visitnitikija. (2551 B.E.). Model of storage inventory efficiency of air conditioning industry, Dissertation of Doctor of Philosophy, Patumthani University.

[5] Cozzolino Alessandra, Humanitarian Logistics and Supply Chain Management, In Humanitarian Logistics, Springer Berlin Heidelberg 2012

[6] Tavatchai Sukanont. (2558 B.E.). Work efficiency of personnel at ACK International Co., Ltd. Master of Business Administration, Kasem Bundit University.

[7] Nuruemon Sanjaivong. (2558 B.E.). Factors affecting consumer choosing International air 